\title{
Fractional spaces and conservation laws
}

\author{
Pierre CASTELLI, Pierre-Emmanuel JABIN, Stéphane JUNCA
}

\begin{abstract}
In 1994, Lions, Perthame and Tadmor conjectured the maximal smoothing effect for multidimensional scalar conservation laws in Sobolev spaces. For strictly smooth convex flux and the one-dimensional case we detail the proof of this conjecture in the framework of Sobolev fractional spaces $W^{s, 1}$, and in fractional $B V$ spaces: $B V^{s}$. The $B V^{s}$ smoothing effect is more precise and optimal. It implies the optimal Sobolev smoothing effect in $W^{s, 1}$ and also in $W^{s, p}$ with the optimal $p=1 / s$. Moreover, the proof expounded does not use the Lax-Oleinik formula but a generalized one-sided Oleinik condition.
\end{abstract}

\section{Introduction}

In this short note we first prove a remark on the optimal smoothing effect in Sobolev spaces given by Lions, Perthame and Tadmor [LPT] for strictly convex flux with a power-law type of degeneracy. Then we improve this result in fractional $B V$ spaces: $B V^{s}$. The smoothing effect in $B V^{s}$ with the optimal $s$ yields to the optimal Sobolev regularity in $W^{s, p}$ with the same exponent $s$ and the optimal $p$. This $B V^{s}$ regularity also gives traces property of the entropy solution ([BM, DOW, DR, P1, P2]). We do not use the Lax-Oleinik formula ([E]) as suggested in [LPT] and used in [BGJ, CJ3] but the one-sided Lipschitz condition on the velocity of the entropy solution (2) below. In all the paper, the following one-dimensional scalar conservation law are considered:

P. Castelli

Lycée Audiberti, 63 bd Wilson, 06600, Antibes France, e-mail: Pierre.Castelli@ac-nice.fr

P-E Jabin

CSCAMM and Dept. of Mathematics, University of Maryland, College Park, MD 20742, USA.

e-mail: pjabin@umd.edu

S. Junca

Université Côte d'Azur, Inria, CNRS, LJAD, Nice, France, e-mail: Stephane.JUNCA@unice.fr 


$$
\left\{\begin{array}{c}
\left.\frac{\partial u}{\partial t}+\frac{\partial}{\partial x}(f(u))=0 \text { on } \mathbb{R} \times\right] 0,+\infty[ \\
u(x, 0)=u_{0}(x) \text { on } \mathbb{R}
\end{array},\right.
$$

where the flux $f$ is strictly convex and $f \in C^{1}$, that is to say that the velocity $a(u)=f^{\prime}(u)$ is strictly increasing. Lax ([La]) and Oleinik ([O]) obtained the optimal smoothing effect in $B V$ for an uniformly convex flux: inf $f^{\prime \prime}>0$. The $B V$ regularity is known to be lost if the second derivative of the flux vanishes at only one point ([Ch1]). Nevertheless we can obtain $B V^{s}$ regularity with $0<s<1 . B V^{s}$ spaces are essentially fractional BV spaces with similar properties as for example $B V=B V^{1}$. First applications of such fractional $B V$ spaces for a $2 \times 2$ system are already obtained in [BGJP].

We do not speak about $S B V$ regularity ([AGV2, AD, B]) since this related to an uniform convex flux and the BV smoothing effect. On the other hand there are many other studies of the regularizing effect ([AGV1, BM, Cc, Ja]) which can be interpreted in the framework of fractional $B V$ spaces but are beyond the scope of this short note.

The paper is organized as follow. In Section 2 a one-sided Lipschitz condition is recalled and linked with the degeneracy of the flux. Then, the smoothing effect is stated in $W^{s, p}$ and $B V^{s}$. Section 3 gives a very simple proof of the smoothing effect in $B V^{s}$ and some consequences as the optimal $W^{s, p}$ regularity. We also present in the final Section 4 an alternative proof of the optimal Sobolev regularity in $W^{s, 1}$.

\section{One-sided condition and degeneracy of the flux}

The proof uses the following generalized one-sided Oleinik inequality due to Dafermos ([D]) (see also [Ch2]) under convex condition on the flux and for almost all $x<y$ :

$$
a(u(y, t))-a(u(x, t)) \leq \frac{y-x}{t}
$$

There is a geometric interpretation of Inequality (2). If there is no shock issued from $(x, 0)$ and $(y, 0)$ in the $(x, t)$ plane then $u$ is constant on the two characteristics issued from $(x, 0)$ and $(y, 0)$ and the constant difference of the velocities is less than the constant velocity needed to meet the characteristics at time $t$. Inequality (2) is usually interpreted as an entropy condition: the jump of velocity can only be negative when $y \rightarrow x$. A complete argument can be given with the generalized characteristics of Dafermos ([D]).

Since any strictly $C^{1}$ convex flux can be approached in $C^{1}$ by a sequence of $C^{2}$ uniformly convex flux, inequality (2) is still valid for entropy solutions almost everywhere. If the flux is uniformly convex then $m|u-v| \leq|a(u)-a(v)|$ for a positive constant $m$ and Inequality (2) yields to the one-sided Oleinik condition: 
$u(y, t)-u(x, t) \leq \frac{y-x}{m t}$ which can be rewritten with $(v)_{+}=\max (v, 0)$ for almost all $(x, y) \in \mathbb{R}^{2}$ and $t>0$ :

$$
(u(y, t)-u(x, t))_{+} \leq \frac{(y-x)_{+}}{m t}
$$

For a non uniformly convex flux, the previous one-sided Lipschitz condition becomes a one-sided Hölder condition: Inequality (5) at the end of this section. Before, a precise definition of a nonlinear flux is needed.

A power-law type of nonlinear degeneracy is considered as in [BGJ]. The following condition is enough for a strictly convex flux with some regularity and powerlaw flux for instances.

Definition 1. Let $f \in C^{1}(K, \mathbb{R})$, where $K$ is a closed interval of $\mathbb{R}$. We say that the degeneracy of $f$ on $K$ is at least $q>0$ if the continuous derivative $a(u)=f^{\prime}(u)$ satisfies :

$$
\inf _{(u, v) \in(K \times K) \backslash \mathscr{D}_{K}} \frac{|a(u)-a(v)|}{|u-v|^{q}}>0,
$$

where $\mathscr{D}_{K}=\{(u, v) \in(K \times K) \mid u \neq v\}$. The lowest real number $q$, if there exists, is called the degeneracy of $f$ on $K$ and denoted $p$.

Inequality 3 is equivalent to:

$$
\exists m>0, \forall(u, v) \in K^{2},|a(u)-a(v)| \geq m|u-v|^{q} .
$$

In particular, (4) implies that $a: K \rightarrow \mathbb{R}$ is strictly monotonic, since $a$ is injective and continuous.

Example 1. If $f(u)=|u|^{1+p}, p \geq 1$, then $p$ is the degeneracy of $f$ on any interval which contains 0 .

Remark 1. 1) Suppose that for all $u, v \in K,|a(u)-a(v)| \geq m|u-v|^{q}$ and that $a$ is differentiable at $u_{0}$. Then $q \geq 1$, since $\infty>\left|\frac{a(u)-a\left(u_{0}\right)}{u-u_{0}}\right| \geq m\left|u-u_{0}\right|^{q-1}$.

2) If $f$ is smooth on $K$, then $f$ has a degeneracy $p$ which is a positive integer. $([\mathrm{BGJ}])$

Replacing $f(u)$ by $-f(-u)$ if necessary, we will assume subsequently that $a(u)=$ $f^{\prime}(u)$ is strictly increasing, so $f$ is strictly convex.

Now, one-sided condition (2) on the velocity is interpreted as the following onesided Hölder condition for almost all $x, y$ :

$$
(u(y, t)-u(x, t))_{+} \leq K \frac{\left[(y-x)_{+}\right]^{s}}{t^{s}},
$$

where $s=\frac{1}{p}, K=m^{-s},(v)_{+}=\max (v, 0)$ since $m(u-v)^{p}=m\left((u-v)_{+}\right)^{p} \leq a(u)-$ $a(v)$ for $u \geq v$. Again, the exponent $s$ naturally appears to estimate the positive variation of the entropy solution $u$.

Now, we can state the smoothing effect for entropy solutions. 
Theorem 1. Let $u_{0}$ belongs to $L^{\infty}(\mathbb{R})$ function, $K$ be the convex hull of $u_{0}(\mathbb{R}), f$ a $C^{1}$ strictly convex flux with degeneracy $p$ on $K$. Then the associated entropy solution of the conservation law (1) have got the following regularity in space for all positive time $t$ and for all $\varepsilon>0: u(., t) \in W_{l o c}^{s-\varepsilon, 1 / s}(\mathbb{R}) \cap B V_{l o c}^{s}(\mathbb{R})$.

The regularity in $W_{l o c}^{s-\varepsilon, 1}(\mathbb{R})$ was conjectured in [LPT] and proved in [Ja]. The regularity in $B V^{s}$ was first proved in [BGJ] . The regularity in $W_{l o c}^{s-\varepsilon, 1 / s}$ is the consequence of the $B V_{l o c}^{s}$ regularity. The optimality in Sobolev spaces can be found in [DW] and in the fractional $B V$ spaces in [CJ1].

The main originality of this note is hence to give new and simpler proofs for this smoothing effect. Our proofs are based on the key $B V$ estimate of the velocity $a(u)$ for positive time given by (2). This is obviously a nonlinear regularity estimate on the entropy solution $u$ which can easily be translated into more traditional regularity estimates.

The spaces $B V^{s}$ are in particular well adapted for this, leading to a very simple proof in the next section. From this $B V^{s}$ regularity it is straightforward to deduce fractional Sobolev regularity as well. But of course one can also prove directly the Sobolev regularity; we give an example of such a proof in the last Section.

\section{$3 B V^{s}$ smoothing effect}

We can define $B V_{+}^{s}$ as $W_{+}^{s, 1}$ and try to adapt the previous proof from Section 4.3. Unfortunately, the equality: $L^{\infty} \cap B V_{+}^{s}=B V^{s}$ for $0<s<1$ is an open problem. It is only known for $s=1$ and, fortunately, it is enough to get the optimal $B V^{s}$ regularity.

\section{$3.1 B V^{s}$ spaces}

We recall briefly the definition and the main properties of fractional $B V$ spaces.

Definition 2. Let $I$ be an non-empty interval of $\mathbb{R}$ and let $\mathscr{S}(I)$ be the set of subdivisions of $I: \mathscr{S}(I)=\left\{\left(x_{0}, x_{1}, \ldots, x_{n}\right), n \geq 1, x_{i} \in I, x_{0}<x_{1}<\ldots<x_{n}\right\}$.

For $0<s \leq 1$ set

$$
T V^{s} u[I]=\sup _{\mathscr{S}(I)} \sum_{i=1}^{n}\left|u\left(x_{i}\right)-u\left(x_{i-1}\right)\right|^{\frac{1}{s}},
$$

then $B V^{s}(I):=\left\{u, T V^{s} u[I]<\infty\right\}$.

For $s=1$ we recover the space $B V$ of functions of bounded variation. Functions in $B V^{s}$ have always left and right traces, like in $B V$ ([MO]). The exponent $s$ is related to the fractional Sobolev derivative: $B V^{s} \subset W^{s-\varepsilon, 1 / s}$ for all $\varepsilon>0$ ([BGJ]). The space $B V^{s}$ is also called the space of functions of bounded $p$-variation, with $p=\frac{1}{s}$. 


\subsection{The short proof of the $B V^{s}$ smoothing}

Set $T V_{+} u:=\sup _{\substack{n \in \mathbb{N}^{*} \\ x_{0}<x_{1}<\ldots<x_{n}}} \sum_{i=1}^{n}\left(u\left(x_{i}\right)-u\left(x_{i-1}\right)\right)_{+}$and $B V_{+}:=\left\{u, T V_{+} u<\infty\right\}$

then $a(u(., t)) \in B V_{+}$from inequality (2). According to the Maximum principle $a(u(x, t)) \in L^{\infty}$ as the initial data $u_{0}$. Since $B V_{+} \cap L^{\infty}=B V$, then $a(u(., t)) \in B V$. Moreover, the velocity $a()$ has at most a power law degeneracy:

$$
|a(u)-a(v)| \geq m|u-v|^{p}
$$

thus $u \in B V^{s}$, where $s=\frac{1}{p}$.

This proof is very short and shortens the proof given in [BGJ]. Moreover, it gives more information about the singularity of $u$. For instance if the flux is a convex power law $f(u)=|u|^{1+p}$ and the convex hull $\mathrm{K}$ of $u_{0}(\mathbb{R})$ does not contain the singular point 0 then the entropy solution associated to the initial date $u_{0}$ belongs to $B V$. That means that the $B V^{s}$ regularity is due to bigger oscillations around the state $u=0$. Notice that since there is only a finite number of oscillations with any given positive strength thus the oscillations near state $u=0$ has to be smaller and smaller and with infinitely many oscillations as optimal examples given in [CJ1, DW].

\section{Optimal smoothing effect in Sobolev spaces $W^{s, 1}$}

The best smoothing effect in Sobolev spaces $W^{s, 1}$ was suggested in [LPT] with the Lax-Oleinik formula, bounded in [DW] and proved in [Ja] with a kinetic formulation and a $B V$ assumption on the velocity. In this short note an another proof is proposed.

We recall a classical result for $W_{+}^{1,1}$ and $B V_{+}$. Then a similar result in $W_{+}^{s, 1}$ is proved and used to get the maximal smoothing effect for conservation laws.

\subsection{Usual results in $W^{1,1}$}

$W_{+}^{1,1}(\mathbb{R})$ is the set of functions $u$ such that the semi-norm $|u|_{1}^{+}=\int_{\mathbb{R}}\left(\partial_{x} u\right)_{+} d x$ is finite, where $(v)_{+}=\max (v, 0)$.

Lemma 1. $W_{+}^{1,1}(\mathbb{R}) \cap L^{1}(\mathbb{R}) \subset B V(\mathbb{R})$.

Notice that $W_{+}^{1,1}(\mathbb{R}) \cap L^{1}(\mathbb{R})$ is bigger than $W^{1,1}(\mathbb{R})$ : for instance consider the $B V$ function $u(x)=x \chi_{[0,1](x)}$ where $\chi_{I}$ is the indicator function of the set $I$.

The lemma follows from the equality $\int_{\mathbb{R}}\left|\partial_{x} u\right| d x=2|u|_{1}^{+}$, which is valid for any smooth compactly supported function, since $|x|=2 x_{+}-x$ and then $\int_{\mathbb{R}}\left|\partial_{x} u\right| d x=$ $2 \int_{\mathbb{R}}\left(\partial_{x} u\right)_{+} d x-\int_{\mathbb{R}} \partial_{x} u d x=2 \int_{\mathbb{R}}\left(\partial_{x} u\right)_{+} d x$. 
The space $B V$ is better fitted through the control of the positive variation since

$$
B V_{+}(\mathbb{R}) \cap L^{\infty}(\mathbb{R})=B V(\mathbb{R})
$$

where $B V_{+}(\mathbb{R})$ is the space of function $u$ such that

$$
T V_{+} u=\sup _{n \in \mathbb{N}, x_{0}<x_{1}<\cdots<x_{n}} \sum_{i=1}^{n}\left(u\left(x_{i}\right)-u\left(x_{i-1}\right)\right)_{+}<\infty .
$$

\subsection{The $W_{+}^{s, 1}(\mathbb{R})$ case}

The usual Sobolev semi-norm in $W^{s, 1}(\mathbb{R})$ for $\left.s \in\right] 0,1[$ is :

$$
|u|_{s}=\int_{\mathbb{R}} \int_{\mathbb{R}} \frac{|u(x)-u(y)|}{|x-y|^{1+s}} d x d y=\iint \frac{|u(x+h)-u(x)|}{|h|^{1+s}} d x d h .
$$

The Sobolev semi-norm in $W_{+}^{s, 1}$ for $\left.s \in\right] 0,1[$ is :

$$
|u|_{s}^{+}=\int_{\mathbb{R}} \int_{h>0} \frac{[u(x+h)-u(x)]_{+}}{|h|^{1+s}} d x d h .
$$

The definition of the set $W_{+}^{s, 1}(\mathbb{R})$ is:

Definition 3. $u \in W_{+}^{s, 1}(\mathbb{R})$ if $\iint_{x>y} \frac{[u(x)-u(y)]_{+}}{|x-y|^{1+s}} d x d y<+\infty$.

Proposition 1. For all $0<s<1, L^{1}(\mathbb{R}) \cap W_{+}^{s, 1}(\mathbb{R})=W^{s, 1}(\mathbb{R})$.

For $s<1$ the estimates for the "one-sided" semi-norm is almost enough to stay in the associated Sobolev space. This is not true for $s=1$ (Lemma 1). Proposition 1 is also valid locally: $L_{\text {loc }}^{1}(\mathbb{R}) \cap W_{+, \text {loc }}^{s, 1}(\mathbb{R})=W_{\text {loc }}^{s, 1}(\mathbb{R})$.

Proof. The bound of the Sobolev semi-norm $2 I=\iint \frac{|u(x)-u(y)|}{|x-y|^{1+s}} d x d y<+\infty$ is obtained thanks to the integral $I=\iint_{x>y} \frac{|u(x)-u(y)|}{|x-y|^{1+s}} d x d y$, which is a priori singular. Consider for $\varepsilon>0$ the well-defined integrals :

$$
\begin{aligned}
& I_{\varepsilon}^{+}=\iint_{x>y} \frac{[u(x)-u(y)]_{+}}{\varepsilon+|x-y|^{1+s}} d x d y \\
& I_{\varepsilon}^{-}=\iint_{x>y} \frac{[u(x)-u(y)]_{-}}{\varepsilon+|x-y|^{1+s}} d x d y
\end{aligned}
$$




$$
\begin{aligned}
& I_{\varepsilon}=\iint_{x>y} \frac{|u(x)-u(y)|}{\varepsilon+|x-y|^{1+s}} d x d y=I_{\varepsilon}^{+}+I_{\varepsilon}^{-} \\
& J_{\varepsilon}=\iint_{x>y} \frac{u(x)-u(y)}{\varepsilon+|x-y|^{1+s}} d x d y=I_{\varepsilon}^{+}-I_{\varepsilon}^{-}
\end{aligned}
$$

Since

$$
\begin{aligned}
J_{\varepsilon} & =\int u(x) \int_{x>y} \frac{1}{\varepsilon+|x-y|^{1+s}} d y d x-\int u(y) \int_{x>y} \frac{1}{\varepsilon+|x-y|^{1+s}} d x d y \\
& =\int u(x) \int_{x>y} \frac{1}{\varepsilon+|x-y|^{1+s}} d y d x-\int u(x) \int_{x<y} \frac{1}{\varepsilon+|x-y|^{1+s}} d y d x, \\
& =\int u(x) \int_{h>0} \frac{1}{\varepsilon+|h|^{1+s}} d h d x-\int u(x) \int_{h<0} \frac{1}{\varepsilon+|h|^{1+s}} d h d x \\
& =0
\end{aligned}
$$

it follows that $I_{\varepsilon}^{+}=I_{\varepsilon}^{-}$and then $I_{\varepsilon}=2 I_{\varepsilon}^{+} \leq 2 I_{0}^{+}<+\infty$. The monotone convergence theorem yields $\lim _{\varepsilon \rightarrow 0} I_{\varepsilon}=I=2 I_{0}^{+}$, so that $u \in W^{s, 1}(\mathbb{R})$.

\subsection{Optimal smoothing effect in fractional Sobolev spaces}

Thanks to Proposition (1), it suffices to get a $W_{+}^{\sigma, 1}$ estimate to have the $W^{\sigma, 1}$ regularity $\forall \sigma<s$. The information is propagated with a finite speed for conservation laws so we work only locally with local Sobolev semi-norm as in [Ju].

The well known embedding from Holder spaces to Sobolev spaces: $W_{l o c}^{s, \infty} \subset$ $W_{l o c}^{\sigma, 1}, \forall \sigma<s$, is simply extended to $W_{+, l o c}^{s, \infty}$ :

Lemma 2. $W_{+, l o c}^{s, \infty} \subset W_{+, l o c}^{\sigma, 1}, \forall \sigma<s$,

Proof. Assume that $(u(x+h)-u(x))_{+} \leq C h^{s}$ for $h>0$.

Let $h_{0}$ and $A$ be some positive constants to estimate the $W_{+, l o c}^{\sigma, 1}$ semi-norm on the interval $]-A, A+h_{0}[$ :

$$
\begin{gathered}
|u|_{\sigma, l o c}^{+}=\int_{|x|<A} \int_{h_{0}>h>0} \frac{[u(x+h)-u(x)]_{+}}{h^{1+\sigma}} d x d h \leq \int_{|x|<A} \int_{h_{0}>h>0} C h^{s-\sigma-1} d x d h \\
|u|_{\sigma, l o c}^{+} \leq 2 A C \int_{h_{0}>h>0} h^{s-\sigma-1} d h
\end{gathered}
$$

which is finite if and only if $\sigma<s$.

Finally, the one-sided Holder condition for an entropy solution is already given by Inequality (5) and the Sobolev regularity follows since an entropy solution is bounded in $L^{\infty}$ as its initial data and $W_{+, l o c}^{\sigma, 1} \cap L^{\infty} \subset W_{l o c}^{\sigma, 1}$.

Acknowledgements We thanks the support of the project SlowDyn (team leader Bruno Lombard): an interdisciplinary CNRS project based on the LMA (CNRS, UPR 7051, Marseille). P-E 
Jabin acknowledge the support of NSF Grants DMS 1312142 and 1614537 and by NSF Grant RNMS (Ki-Net) 1107444.

\section{References}

[AGV1] Adimurthi, S. S. Ghoshal, G. D. Veerappa Gowda. Structure of entropy solutions to scalar conservation laws with strictly convex flux. J. Hyperbolic Differ. Equ. 9 (2012), no. 4, 571611.

[AGV2] Adimurthi, S. S. Ghoshal, G. D. Veerappa Gowda. Finer regularity of an entropy solution for 1-d scalar conservation laws with non uniform convex flux. Rend. Semin. Mat. Univ. Padova 132 (2014), 124.

[AD] L. Ambrosio, C. De Lellis. A note on admissible solutions of $1 D$ scalar conservation laws and 2D Hamilton-Jacobi equations. J. Hyperbolic Differ. Equ. 1 (2004), no. 4, 813826.

[B] S.Bianchini. SBV regularity for scalar conservation laws. Hyperbolic problemstheory, numerics and applications. Volume 1, 311, Ser. Contemp. Appl. Math. CAM, 17, World Sci. Publishing, Singapore, 2012.

[BM] S. Bianchini, E. Marconi. On the structure of $L^{\infty}$-entropy solutions to scalar conservation laws in one-space dimension. Preprint SISSA 43/2016/MATE.

[BGJ] C. Bourdarias, M. Gisclon, S. Junca. Fractional BV spaces and applications to scalar conservation laws. J. Hyperbolic Differential Equations, 11, 4, (2014), 655-677.

[BGJP] C. Bourdarias, M. Gisclon, S. Junca, Y.-J Peng. Eulerian and Lagrangian formulations in $B V^{s}$ for gas-solid chromatography. Commun. Math. Sci. 14 (2016), no. 6, 1665-1685.

[CJ1] P. Castelli, S. Junca. Oscillating waves and the maximal smoothing effect for onedimensional nonlinear conservation laws. AIMS Ser. on Appl. Math. 8, (2014), 709-716.

[CJ2] P. Castelli, S. Junca. On the maximal smoothing effect for multidimensional scalar conservation laws. Preprint: hal-01290871, (2016), 14 pp.

[CJ3] P. Castelli, S. Junca. Smoothing effect in $B V-\Phi$ for scalar conservation laws. Preprint: hal-01133725, version 2, (2016), $30 \mathrm{pp}$.

[Ch1] K. S. Cheng. The space BV is not enough for hyperbolic conservation laws. J. Math. Anal. App., 91, 2, (1983), 559-561.

[Ch2] K. S. Cheng. A regularity theorem for a nonconvex scalar conservation law. J. Differential Equations 61, no. 1, (1986), 79-127.

[Cc] C. Cheverry. Regularizing effects for multidimensional scalar conservation laws. Ann. Inst. H. Poincar Anal. Non Linaire 17 (2000), no. 4, 413472.

[D] C. Dafermos. Regularity and large time behavior of solutions of a conservation law without convexity. Proc. Royal Soc.Edhinburgh 99 A, (1985), 201-239.

[DOW] C. De Lellis, F. Otto, M. Westdickenberg. Structure of entropy solutions for multidimensional scalar conservation laws. Arch. Ration. Mech. Anal. 170 (2003), no. 2, 137-184.

[DR] C. De Lellis, T. Rivire. The rectifiability of entropy measures in one space dimension. J. Math. Pures Appl. (9) 82 (2003), no. 10, 1343-1367.

[DW] C. De Lellis, M. Westdickenberg. On the optimality of velocity averaging lemmas. Ann. Inst. H. Poincar, Anal. Nonlinaire 20 (2003), no. 6, 1075-1085.

[E] Laurence C. Evans. Partial differential equations. American Mathematical Society, (1998).

[Ja] P.-E. Jabin. Some regularizing methods for transport equations and the regularity of solutions to scalar conservation laws. [2008-2009], Exp. No. XVI, Smin. Equ. Driv. Partielles, Ecole Polytech., Palaiseau, (2010).

[Ju] S. Junca. High frequency waves and the maximal smoothing effect for nonlinear scalar conservation laws. SIAM Math. Anal. 46 (2014), no. 3, 2160-2184.

[La] P. D. Lax. Hyperbolic systems of conservation laws. II. Comm. Pure Appl. Math. (1957), 10, 537-566.

[LPT] P.-L. Lions, B. Perthame, E. Tadmor. A kinetic formulation of multidimensional scalar conservation laws and related equations. J. Amer. Math. Soc. 7, (1994), 169-192. 
[O] O. Oleinik. Discontinuous solutions of nonlinear differential equations. Usp. Mat. Nauk., 12, (1957), 3-73. English transl. in Am. Math. Soc. Transl. Ser. 2 (1963), no. 26, 95-172.

[P1] E.Yu. Panov. Existence of strong traces for generalized solutions of multidimensional scalar conservation laws. J. Hyperbolic Differ. Equ. 2 (2005), no. 4, 885908.

[P2] E.Yu. Panov. Existence of strong traces for quasi-solutions of multidimensional conservation laws. J. Hyperbolic Differ. Equ. 4 (2007), no. 4, 729770.

[MO] J. Musielak, W. Orlicz. On generalized variations. I. Studia Math. 18 (1959), 11-41. 